\title{
Über regulär-singuläre Lösungen von Systemen linearer Differentialgleichungen. II*)
}

\author{
Von Ekkehard Wagenführer in Regensburg
}

Im I. Teil der vorliegenden Arbeit befaßten wir uns mit der formalen Lösbarkeit der Matrix-Differentialgleichung

$$
x^{s+1} Y^{\prime}(x)-B(x) Y(x)=0
$$

durch formale Potenzreihen

Dabei ist

$$
Y(x)=\sum_{\nu=0}^{\infty} H_{\nu} x^{\nu I+J}
$$

$$
\begin{array}{cl}
s \in \mathbb{N}, & R>0, \\
B: \mathfrak{\Re}_{R} \rightarrow M_{n}(\mathbb{C}) & \text { holomorph, } \\
B(x)=\sum_{\nu=0}^{\infty} x^{\nu} B_{v} & \text { für }|x|<R,
\end{array}
$$

vorausgesetzt.

Wir sind davon ausgegangen, daß für die Koeffizienten einer regulär-singulären Lösung von (1) der Form

$$
Y(x)=H(x) x^{J}=\sum_{\nu=0}^{\infty} H_{\nu} x^{\nu I+J}
$$

mit konstantem $J \in M_{n}(\mathbb{C})$ und

$$
H(x)=\sum_{\nu=0}^{\infty} x^{\nu} H_{v} \quad \text { holomorph für } x \in \mathfrak{\Re}_{R}
$$

notwendig die folgenden Gleichungen gelten:

$$
\begin{cases}\sum_{\nu=0}^{\mu} B_{\mu-\nu} H_{\nu}=0 & (\mu=0, \ldots, s-1) \\ \sum_{\nu=0}^{\mu} B_{\mu-\nu} H_{\nu}-H_{\mu-s}(J+(\mu-s) I)=0 & (\mu=s, s+1, \ldots) .\end{cases}
$$

Das algebraische Problem, die Gleichungsfolge (1.2) bei maximalem Rang der formalen Potenzreihe zu lösen, war Inhalt des 1. Kapitels, das den I. Teil unserer Arbeit ausfüllte.

*) Der I. Teil der Arbeit ist in dieser Zeitschrift, Bd. 267 (1974), erschienen. In dem vorliegenden Teil müssen wir an mehreren Stellen auf Formeln und Sätze des I. Teils, die mit (1. . ) numeriert sind, verweisen. 


\section{Konvergente Lösungen}

Im folgenden wollen wir (1. 2) lösen unter der Konvergenzbedingung

$$
\sum_{\nu=0}^{\infty} x^{\nu} H_{\nu} \quad \text { konvergent für } x \in \AA_{R}
$$

dann ist durch die Potenzreihe

$$
Y(x)=\sum_{\nu=0}^{\infty} H_{\nu} x^{\nu I+J}
$$

eine regulär-singuläre Lösung von (1) dargestellt.

2. 1. Reduktion der Konvergenzbedingung. In diesem Unterkapitel wollen wir für $s$ auch den Wert Null zulassen; von $M_{n}(\mathbb{C})$ benutzen wir nur die Eigenschaften als BanachAlgebra. Es bezeichne also

$$
\mathfrak{B}:=M_{n}(\mathbb{C}) ;
$$

als Norm in $M_{n}(\mathbb{C})$ wählen wir für $A=\left(\alpha_{i j}\right)_{(n, n)}$ :

Ferner bezeichne

$$
|A|=\max _{i=1}^{n}\left(\sum_{j=1}^{n}\left|\alpha_{i j}\right|\right) \text {. }
$$

$$
\begin{aligned}
\mathfrak{L}_{1}(\mathfrak{B}) & =\{\mathrm{G} \mid \mathrm{G}: \mathfrak{B} \rightarrow \mathfrak{B} \text { linear (und stetig) }\}, \\
\mathfrak{F} & =\left\{\left(H_{\nu}\right)_{\nu=0}^{\infty} \in \mathfrak{B}^{\mathrm{N}_{0}} \mid \sum_{\nu=0}^{\infty} x^{\nu} H_{\nu} \text { kgt. für } x \in \mathfrak{\Re}_{R}\right\} .
\end{aligned}
$$

Für das Folgende seien

fest vorgegeben.

$$
l>0 \quad \text { sowie } \quad J \in \mathfrak{B} \quad \text { mit }|J| \leqq l
$$

Satz 2. 1. Ab einer natürlichen Zahl $N^{\prime}$, die nur von l abhängt, gilt für alle $N \geqq N^{\prime}$ : $Z u$ beliebig vorgegebenen $H_{0}, \ldots, H_{N-1} \in \mathfrak{B}$ existieren eindeutig $H_{N}, H_{N+1}, \ldots \in \mathfrak{B}$ mit

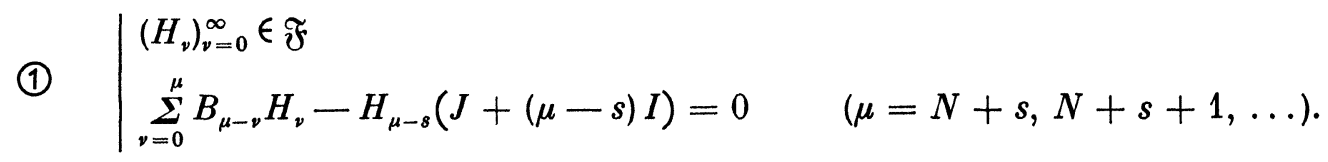

Dazu existieren (von $J$ abhängige)

mit

$$
\boldsymbol{G}_{\mu, \nu} \in \mathfrak{L}_{\mathbf{1}}(\mathfrak{B}) \quad\left(\mu=N^{\prime}, N^{\prime}+1, \ldots ; \nu=0,1, \ldots, \mu\right)
$$

so daß (1) äquivalent $z u$

$$
G_{\mu, \mu}=-I
$$

(2)

$$
\mid \sum_{\nu=0}^{\mu} G_{\mu, \nu} H_{\nu}=0 \quad(\mu=N, N+1, \ldots) .
$$

Zur Erläuterung notieren wir

Folgerung 2. 2. In (2) sind die Werte $H_{N}, H_{N+1}, \ldots$ schon gegeben durch

$$
H_{\mu}=\sum_{\nu=0}^{\mu-1} G_{\mu \nu} H_{\nu} \quad(\mu=N, N+1, \ldots),
$$

also liegen alle Lösungsfolgen von (2) bzw. (2. 3) in $\mathfrak{F}$. 
Dem Beweis des Satzes ist das folgende Unterkapitel gewidmet, zunächst beschäftigen wir uns mit einer Verallgemeinerung des $l_{\infty}$. gemein

2. 1. 1. Beweis von Satz 2. 1. Für komplexe Banach-Räume $\mathfrak{R}, \mathfrak{S}$ bezeichne all-

$$
\begin{aligned}
\mathfrak{L}_{\mathbf{1}}(\mathfrak{R}, \mathfrak{S}) & =\{\Lambda \mid \Lambda: \mathfrak{R} \rightarrow \mathfrak{S} \text { stetig, linear }\}, \\
\mathfrak{l}_{\mathbf{1}}(\mathfrak{R}) & =\mathfrak{L}_{\mathbf{1}}(\mathfrak{R}, \mathfrak{R}) .
\end{aligned}
$$

Für $0<R^{\prime}<R$ definieren wir

Mit der Norm

$$
\mathfrak{F}_{R^{\prime}}=\left\{\mathrm{X}=\left(H_{v}\right)_{v=0}^{\infty} \in \mathfrak{B}^{\mathbb{N}_{0}} \mid \sup _{v \in \mathbb{N}_{0}}\left(R^{\prime v}\left|H_{v}\right|\right)<\infty\right\}
$$

$$
|\mathrm{X}|_{R^{\prime}}=\sup _{v \in \mathbb{N}_{0}}\left(R^{\prime v}\left|H_{v}\right|\right)
$$

ist $\mathfrak{F}_{R^{\prime}}$ bekanntlich Banach-Raum; mit $|\Lambda|_{R^{\prime}}$ sei auch die Operatornorm für $\Lambda \in \mathfrak{Q}_{\mathbf{1}}\left(\mathfrak{F}_{R^{\prime}}\right)$ bezeichnet.

Die Koeffizientenabschätzung für Potenzreihen liefert

$$
X \in \mathfrak{F} \curvearrowleft \forall R^{\prime} \quad\left(0<R^{\prime}<R\right) \quad X \in \mathfrak{F}_{R^{\prime}}
$$

Falls $\mathrm{X}=\left(H_{v}\right)_{\nu=0}^{\infty} \in \mathfrak{F}_{R^{\prime}}, A \in \mathfrak{B}$, definieren wir

$$
\mathrm{X} A:=\left(H_{v} A\right)_{\nu=0}^{\infty} .
$$

Vermöge (2.5) entspricht $X$ einer linearen, beschränkten Abbildung von $\mathfrak{B}$ in $\mathfrak{F}_{R^{\prime}}$, die wir auch $X$ nennen.

Eine Verallgemeinerung unendlicher Matrizen liefert uns

Hilfssatz 2. 6. Es seien $L_{\mu \nu} \in \mathfrak{L}_{1}(\mathfrak{B})\left(\mu, \nu \in \mathbb{N}_{0}\right)$ mit

Dann gilt

$$
\sup \left\{R^{\prime \mu} \sum_{\nu=0}^{j} \frac{\left|\boldsymbol{L}_{\mu \nu}\right|}{R^{\prime \nu}}: \mu, j \in \mathbb{N}_{0}\right\}=: K<\infty .
$$

a)

$$
\mathrm{X}=\left(H_{v}\right)_{v=0}^{\infty} \in \mathfrak{F}_{R^{\prime}} \curvearrowright \forall \mu \in \mathbb{N}_{0} \sum_{\nu=0}^{\infty} L_{\mu \nu} H_{\nu} \quad \text { absolut kgt. }
$$

Definiert man

so ist

$$
\Lambda \mathrm{X}:=\left(\sum_{\nu=0}^{\infty} \mathrm{L}_{\mu \nu} H_{\nu}\right)_{\mu=0}^{\infty}
$$

b)

$$
\Lambda \in \mathfrak{Q}_{\mathbf{1}}\left(\mathfrak{F}_{R^{\prime}}\right), \quad|\Lambda|_{R^{\prime}} \leqq K
$$

Wir schreiben kurz

$$
\Lambda=\left(\boldsymbol{L}_{\mu \nu}\right)_{\mu, \nu \in \mathrm{N}_{0}} \cdot
$$

Beweis. Die Voraussetzung liefert uns, daß für jedes $\mu \in \mathbb{N}_{0}$

daher

$$
\sum_{\nu=0}^{\infty} \frac{\left|\boldsymbol{L}_{\mu \nu}\right|}{R^{\prime \nu}}<\infty
$$

$$
\sum_{\nu=0}^{\infty}\left|L_{\mu \nu} H_{\nu}\right| \leqq \sum_{\nu=0}^{\infty} \frac{\left|L_{\mu \nu}\right|}{R^{\prime \nu}}\left(R^{\prime \nu}\left|H_{\nu}\right|\right) \leqq|X|_{R^{\prime}} \cdot \sum_{\nu=0}^{\infty} \frac{\left|L_{\mu \nu}\right|}{R^{\prime \nu}}
$$


Der Ausdruck $\sum_{\nu=0}^{\infty} L_{\mu \nu} H_{\nu}$ ist damit als Grenzwert in $\mathfrak{B}$ erklärt, und für jedes $\mu \in \mathbb{N}_{0}$ gilt die Abschätzung

$$
R^{\prime \mu}\left|\sum_{\nu=0}^{\infty} L_{\mu \nu} H_{\nu}\right| \leqq R^{\prime \mu} \sum_{\nu=0}^{\infty}\left|\frac{L_{\mu \nu}}{R^{\prime \nu}}\right| \cdot|X|_{R^{\prime}} \leqq K \cdot|X|_{R^{\prime}}
$$

womit auch die zweite Behauptung folgt.

Zur Anwendung dieser Überlegungen formen wir bei beliebigem $N \in \mathbb{N}$ die Rekursionen unter Satz 2. 1, (1) geeignet um. Durch Trennen der Summen und Verschieben der Indizes $\mu$ erhalten wir als äquivalente Gleichungen

$$
H_{\mu}(J+\mu I)-\sum_{\nu=N}^{\mu+s} B_{\mu+s-\nu} H_{\nu}=\sum_{\nu=0}^{N-1} B_{\mu+s-\nu} H_{\nu} \quad(\mu=N, N+1, \ldots) .
$$

Wir definieren die Operatoren $L_{v} \in \mathfrak{L}_{1}(\mathfrak{B})$ durch

$$
\left.\begin{array}{l}
\mathbf{L}_{v} A:=B_{v} \cdot A \quad(v \neq s) \\
\boldsymbol{L}_{s} A:=B_{s} A-A J
\end{array}\right\}(A \in \mathfrak{B}) .
$$

Wegen $|\boldsymbol{I}|=1$ gilt für die Operatornormen der $\boldsymbol{L}_{v}$

$$
\begin{aligned}
& \left|\boldsymbol{L}_{\nu}\right|=\left|B_{\nu}\right| \quad(\boldsymbol{v} \neq s) \\
& \left|\boldsymbol{L}_{s}\right| \leqq\left|B_{s}\right|+|J| \leqq\left|B_{s}\right|+l .
\end{aligned}
$$

Ersetzt man $\mu$ durch $N+\mu$, wird (2.7) nach Division durch $N+\mu$ zu

$$
\begin{aligned}
H_{N+\mu}-\frac{1}{N+\mu} \sum_{\nu=0}^{\mu+8} L_{\mu+s-\nu} H_{N+\nu}=\frac{1}{N+\mu} \sum_{\nu=0}^{N-1} B_{N+\mu+8-\nu} H_{\nu} \\
(\mu=0,1,2, \ldots) .
\end{aligned}
$$

Für $N \in \mathbb{N}$ bezeichne

$$
\begin{aligned}
\Phi_{N \nu} & =\left(\frac{1}{N+\mu} B_{N+\mu+s-v}\right)_{\mu=0}^{\infty} & & (v=0,1, \ldots, N-1), \\
\mathrm{X}_{N} & =\left(H_{N+\nu}\right)_{v=0}^{\infty}, & & \text { mit } L_{v}=0 \text { für } v \leqq-1 .
\end{aligned}
$$

$\mathrm{Daß}$ wir System (2.8) umformen können zu

$$
\left(\mathrm{E}-\Lambda_{N}\right) \mathrm{X}_{N}=\sum_{\nu=0}^{N-1} \Phi_{N v} H_{v} \quad\left(\mathrm{E}=\text { Identität in } \mathfrak{F}_{R^{\prime}}\right)
$$

rechtfertigen wir durch

Hilfssatz 2. 10. 1) $\forall N \in \mathbb{N}, v=0,1, \ldots, N-1$ gilt $\Phi_{N v} \in \mathfrak{F}$

2) Für jedes $R^{\prime}$ mit $0<R^{\prime}<R$ gilt
a) $\Phi_{N v} \in \mathfrak{L}_{1}\left(\mathfrak{B}, \mathfrak{F}_{R^{\prime}}\right)$ gemä $\beta(2.5)$
$(N \in \mathbb{N}, v=0,1, \ldots, N-1)$,
b) $\Lambda_{N} \in \mathfrak{Q}_{1}\left(\mathfrak{F}_{R^{\prime}}\right)$ im Sinne von Hilfssatz $2.6 \quad(N \in \mathbb{N})$,
c) $\left|\Lambda_{N}\right|_{R^{\prime}} \rightarrow 0$
$(N \rightarrow \infty)$ gleichmäßig für $|J| \leqq l$.
d) Es existiert $N^{\prime}\left(l, R^{\prime}\right) \in \mathbb{N}$, so daß, für alle $N \geqq N^{\prime}\left(l, R^{\prime}\right), \mathrm{E}-\Lambda_{N}$ invertierbar in $\mathfrak{Q}_{1}\left(\mathfrak{F}_{R^{\prime}}\right)$.


Aus der Holomorphie von $B(x)$ für $x \in \mathfrak{R}_{R}$ folgt die 1. Aussage und mit (2.4) auch $2)$ a). Weiter sei $R^{\prime \prime}$ mit $R^{\prime}<R^{\prime \prime}<R$ gewählt; es bezeichne

$$
\vartheta=\frac{R^{\prime}}{R^{\prime \prime}}, \quad M=\max \left\{|B(x)||| x \mid \leqq R^{\prime \prime}\right\}
$$

Mit Koeffizientenabschätzung nach Cauchy folgt:

$$
\left|\mathbf{L}_{v}\right| \leqq \vartheta^{v} \frac{M}{R^{\prime \nu}} \quad(v \neq s), \quad\left|L_{s}\right| \leqq l+\vartheta^{s} \frac{M}{R^{\prime s}}
$$

Wir verifizieren für $\Lambda_{N}$ die Voraussetzung von Hilfssatz 2.6, wobei in der $\mu$-ten Zeile jeweils nur bis $\mu+s$ zu summieren ist; für jedes $\mu \in \mathbb{N}_{0}$ :

daher

$$
\begin{aligned}
R^{\prime \mu} \cdot \frac{1}{N+\mu} \sum_{\nu=0}^{\mu+8} \frac{\left|L_{\mu+s-\nu}\right|}{R^{\prime \nu}} & \leqq \frac{1}{N+\mu}\left(M \cdot R^{\prime \mu} \sum_{\nu=0}^{\mu+8} \frac{\vartheta^{\mu+s-\nu}}{R^{\prime \mu+s}}+l\right) \\
& \leqq \frac{1}{N+\mu}\left(\frac{M}{(1-\vartheta) R^{\prime s}}+l\right) ;
\end{aligned}
$$

$$
\sup _{\mu \in \mathbb{N}_{0}}\left\{R^{\prime \mu} \cdot \frac{1}{N+\mu} \sum_{\nu=0}^{\mu+8} \frac{\left|L_{\mu+s-\nu}\right|}{R^{\prime \nu}}\right\} \leqq \frac{1}{N}\left(\frac{M}{(1-\vartheta) R^{\prime s}}+l\right) .
$$

Anwendung von Hilfssatz 2. 6 liefert unmittelbar die Aussagen 2), b) und c). Wählt man $N^{\prime}\left(l, R^{\prime}\right)$, so daß

$$
\forall N \geqq N^{\prime}\left(l, R^{\prime}\right) \quad\left|\Lambda_{N}\right|_{R^{\prime}} \leqq \frac{1}{N}\left(\frac{M}{(1-\vartheta) R^{\prime s}}+l\right)<1,
$$

so ist nach dem bekannten Satz über die Neumannsche Reihe auch die letzte Behauptung erfüllt.

Für $N \geqq N^{\prime}\left(l, R^{\prime}\right)$ ist, bei beliebig vorgegebenen $H_{0}, \ldots, H_{N-1} \in \mathfrak{B}$, (2.9) eindeutig nach $X_{N}$ auflösbar, wobei

$$
\mathrm{X}_{N}=\sum_{\nu=0}^{N-1}\left(\mathrm{E}-\Lambda_{N}\right)^{-1} \Phi_{N \nu} H_{\nu}
$$

Weil selbstverständlich

$$
\mathrm{X}_{N} \in \mathfrak{F}_{R^{\prime}} \curvearrowright\left(H_{v}\right)_{v=0}^{\infty} \in \mathfrak{F}_{R^{\prime}}
$$

können wir zusammenfassend notieren:

Unter den Voraussetzungen

$$
\mid \begin{aligned}
& 0<R^{\prime}<R ; \quad l>0,|J| \leqq l \\
& \left.N \geqq N^{\prime}\left(l, R^{\prime}\right) \quad \operatorname{nach}(2.10), \mathrm{d}\right) \\
& H_{0}, \ldots, H_{N-1} \in \mathfrak{B}
\end{aligned}
$$

gilt

Hilfsatz 2. 13. Es existiert genau eine Folge $\mathrm{X}_{N}=\left(H_{\nu}\right)_{\nu=N}^{\infty}$ mit der durch $N$ und $R^{\prime}$ charakterisierten Eigenschaft

$$
\&\left(N, R^{\prime}\right)\left\{\begin{array}{l}
\sum_{\nu=0}^{\mu} B_{\mu-\nu} H_{\nu}-H_{\mu-s}(J+(\mu-s) I)=0 \quad(\mu=N+s, N+s+1, \ldots) \\
\sup _{\nu \in \mathbb{N}_{\bullet}}\left(R^{\prime \nu}\left|H_{\nu}\right|\right)<\infty .
\end{array}\right.
$$


Zum Beweis von Satz 2. 1, (1) fehlt, daß in jedem Fall schon

$$
\mathrm{X}_{N} \in \mathfrak{F} \text {. }
$$

Ist (2.14) bewiesen, wählt man in Satz 2.1

$$
N^{\prime}=\min _{0<R^{\prime}<R} N^{\prime}\left(l, R^{\prime}\right) \text {. }
$$

Als Hilfsmittel dient folgende Überlegung zu Hilfssatz 2. 13:

Korollar 2. 15. Unter Voraussetzung (2.12) nehmen wir $N_{1}>N$ an, zu $H_{0}, \ldots, H_{N-1}$ wählen wir die Glieder $H_{N}, \ldots, H_{N_{1}-1}$ der Folge $\mathrm{X}_{N}$ hinzu.

Anwendung von Hilfsatz 2.13 auf $N_{1}$ statt $N$ liefert eine eindeutig bestimmte Folge

$$
\hat{\mathrm{X}}_{N_{1}}=\left(\hat{H}_{v}\right)_{v=N_{1}}^{\infty} \quad \text { mit } \&\left(N_{1}, R^{\prime}\right) \text {. }
$$

Letztere Eigenschaft ist auch für den Folgenabschnitt

$$
\mathrm{X}_{N_{1}}=\left(H_{v}\right)_{\nu=N_{1}}^{\infty}
$$

von $\mathrm{X}_{N}$ erfüllt. Auf Grund der Eindeutigkeit ist

$$
\hat{H}_{v}=H_{v} \quad \text { für alle } \nu \geqq N_{1} .
$$

$\mathrm{Zu}$ (2.14) müssen wir zeigen, daß für beliebiges $R^{\prime \prime}$ mit $R^{\prime}<R^{\prime \prime}<R$ :

$$
\mathrm{X}_{N} \in \mathfrak{F}_{R^{\prime \prime}}
$$

Hilfssatz 2.13 wenden wir an auf $R^{\prime \prime}$, ein $N_{1}$ mit

$$
N_{1}>\max \left(N, N^{\prime}\left(l, R^{\prime \prime}\right)\right)
$$

sowie $H_{0}, \ldots, H_{N_{1}-1}$. Es ergibt sich eindeutig eine Folge

$$
\hat{\mathrm{X}}_{N_{1}}=\left(\hat{H}_{v}\right)_{v=N_{1}}^{\infty} \quad \text { mit } \&\left(N_{1}, R^{\prime \prime}\right),
$$

die wegen $R^{\prime}<R^{\prime \prime}$ auch $\&\left(N_{1}, R^{\prime}\right)$ erfüllt. Aus Korollar (2.15) folgt, daß

und daher

$$
\hat{\mathrm{X}}_{N_{1}}=\left(H_{v}\right)_{v=N_{1}}^{\infty}
$$

$$
\mathrm{X}_{N} \in \mathfrak{F}_{R^{\prime \prime}}
$$

Ähnlich zeigen wir die Äquivalenz von (1) und (2) in Satz 2. 1. Es sei $R^{\prime}$ mit $N^{\prime}\left(l, R^{\prime}\right)=N^{\prime}$ gewählt und $N \geqq N^{\prime}$. Dann ist für $\mathrm{X}_{N}=\left(H_{v}\right)_{v=N}^{\infty}$ Aussage (1) damit gleichbedeutend, daß $\mathrm{X}_{N}$ Lösung von (2.11) mit $\mathrm{X}_{N} \in \mathfrak{F}_{R^{\prime}}$. Bezeichnet man mit

die Zuordnung

$$
p_{0}: \mathfrak{F}_{R^{\prime}} \rightarrow \mathfrak{B}
$$

$$
\left(H_{v}\right)_{v=0}^{\infty} \mapsto H_{0} \quad \text { und } \quad G_{N v}:=p_{0} \circ\left(\mathrm{E}-\Lambda_{N}\right)^{-1} \circ \Phi_{N v} \quad\left(N \geqq N^{\prime}, v=0, \ldots, N-1\right),
$$

ergibt sich $H_{N}$, die erste Komponente von $\mathrm{X}_{N}$, als

$$
H_{N}=\sum_{\nu=0}^{N-1} G_{N}, H_{\nu} \quad \text { mit } G_{N \nu} \in \mathfrak{L}_{1}(\mathfrak{B}) .
$$

Nach Karollar 2.15 berechnet sich $\mathrm{X}_{N+1}=\left(H_{v}\right)_{v=N+1}^{\infty}$ durch

$$
\mathrm{X}_{N+1}=\sum_{\nu=0}^{N}\left(\mathrm{E}-\Lambda_{N+1}\right)^{-1}\left(\Phi_{N+1, \nu} H_{v}\right),
$$


folglich

$$
H_{N+1}=\sum_{\nu=0}^{N} G_{N+1 v} H_{\nu}
$$

Wiederholte Anwendung von Korollar (2.15) liefert also (2.3) und damit (2) als unendliches Gleichungssystem für $\left(H_{v}\right)_{v=N}^{\infty}$. Die Äquivalenz von (1) und (2) folgt aus der schon im Algebraischen eindeutigen Lösbarkeit von (2).

2. 1. 2. Rückführung aư ein endliches Gleichungssystem. In Satz 2.1 sind die Rekursionen (1.2) nur ab $\mu=N+s$ berücksichtigt, die restlichen liefern weitere Bedingungen für $H_{0}, \ldots, H_{N+8-1}$. Im Anschluß an Satz 2. 1, mit

notieren wir

$$
|J| \leqq l, N \geqq N^{\prime}
$$

Satz 2. 16. Die Reihe $Y(x)=\sum_{\nu=0}^{\infty} H_{\nu} x^{\nu I+J}$ ist konvergente Lösung der Differentialgleichung (1) genau dann, wenn für $H_{0}, \ldots, H_{\mathrm{N}+8-1}$ gilt:

$$
\begin{cases}\text { a) } \sum_{\nu=0}^{\mu} B_{\mu-\nu} H_{\nu}=0 & (\mu=0, \ldots, s-1) \\ \text { b) } \sum_{\nu=0}^{\mu} B_{\mu-\nu} H_{\nu}-H_{\mu-s}(J+(\mu-s) I)=0 & (\mu=s, \ldots, N+s-1) \\ \text { c) } \sum_{\nu=0}^{\mu} G_{\mu \nu} H_{\nu}=0 & (\mu=N, \ldots, N+s-1) .\end{cases}
$$

Die weiteren Koeffizienten $H_{N+8}, H_{N+8+1}, \ldots$ sind durch

$$
H_{\mu}=\sum_{\nu=0}^{\mu-1} G_{\mu \nu} H_{\nu} \quad(\mu=N+s, N+s+1, \ldots)
$$

eindeutig bestimmt.

Der Beweis liegt in einer Zusammenfassung bisheriger Überlegungen, wonach $Y$ Lösung von (1) genau dann, wenn

$$
\left(H_{v}\right)_{\nu=0}^{\infty} \text { Lösung von (1.2) und }\left(H_{v}\right)_{\nu=0}^{\infty} \in \mathfrak{F} .
$$

Dabei stehen die Gleichungen (1.2) für $\mu=0, \ldots, N+s-1$ unter (2.17) a), b), und nach Satz 2.1 ist (1.2) für $\mu=N+s, N+s+1, \ldots$ zusammen mit der Bedingung, $\operatorname{da} \beta\left(H_{v}\right)_{v=0}^{\infty} \in \mathfrak{F}$, gleichbedeutend mit (2.17) c) zusammen mit (2.18).

Da wir $M_{n}(\mathbb{C})$ in diesem Kapitel bisher nur als Banach-Algebra benutzt haben, bleibt Satz 2.16 in jeder komplexen Banach-Algebra $\mathfrak{B}$ gültig. Im Fall $s=0$ reduziert sich (2.17) auf (2.17) b), woraus wir ersehen, daß jede formale Lösung von (1) auch schon konvergiert.

Die folgenden Untersuchungen beziehen sich wieder auf den Fall $s>0$, unter Benutzung der speziellen algebraischen Eigenschaften von $M_{n}(\mathbb{C})$.

2. 2. Lösung des reduzierten Problems. Unser Ziel sind Kriterien zur nichttrivialen Lösbarkeit von (2.17), ohne die $G_{\mu \nu}$ explizit zu kennen. Eine Lösung

$$
Y(x)=\sum_{\nu=0}^{\infty} H_{\nu} x^{\nu I+J}
$$


von (1) ist notwendig formale Lösung, als deren Rang wir nicht unbedingt $r_{\max }$ annehmen dürfen. Nach Kapitel 1 setzen wir

$\mid J$ in Gestalt (1.4) mit $r>0$

wobei wir nach den Überlegungen zu Satz 1. 32

$\mid \begin{aligned} & \lambda_{1}, \ldots, \lambda_{m} \text { als (hier im Unterschied } z u \text { Satz } 1.32 \text { nicht notwendig alle) Nullstellen } \\ & \text { von } \chi \text { mit } \chi\left(\lambda_{i}-z\right) \neq 0 \text { für alle } z \in \mathbb{N}\end{aligned}$

annehmen. Ferner sei

$\mid p=$ maximale ganzzahlige Differenz von Nullstellen von $\chi ;$

schließlich wählen wir zur Anwendbarkeit von Satz 2. 1 und damit Satz 2. 16

$$
\mid \begin{aligned}
& l>\max \{|\lambda| \mid \lambda \text { Nullstelle oon } \chi\}+1, \\
& N \geqq \max \left(N^{\prime}, p+q+2-s\right)
\end{aligned}
$$

mit $q$ aus (1.24), so daß

$$
\left\{\begin{array}{l}
|J|<l \\
N+s-1 \geqq p+q+1 .
\end{array}\right.
$$

Unter dieser Voraussetzung untersuchen wir die $G_{\mu \nu}$ auf invariante lineare Unterräume von $M_{n}(\mathbb{C})$. Es sei

$$
J_{i}^{\tau} \text { ein festes Jordankästchen von } J \text {, und kurz } k:=k_{i}^{\tau} \text { gesetzt. }
$$

Dann definieren wir die Projektionen

durch

$$
\boldsymbol{P}_{x} \in \mathfrak{R}_{1}\left(M_{n}(\mathbb{C})\right) \quad(\varkappa=1, \ldots, k)
$$

mit den Matrizen

$$
P_{x} A:=A \cdot P_{x} \quad \text { für } A \in M_{n}(\mathbb{C})
$$

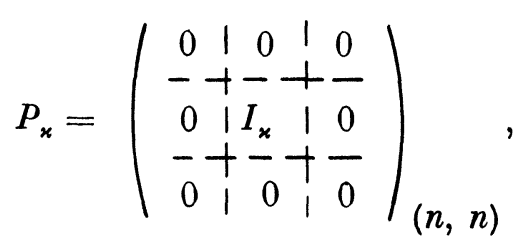

$I_{x}$ an der Stelle von $J_{i}^{\tau}$,

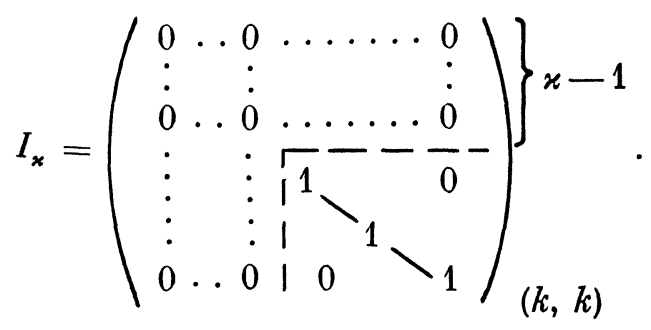

Weiter bezeichne

$$
\mathfrak{u}_{\kappa}=\boldsymbol{P}_{x}\left(M_{n}(\mathbb{C})\right) \quad(\varkappa=1, \ldots, k) .
$$

Dann ist $\mathfrak{H}_{x}$ linearer Unterraum von $M_{n}(\mathbb{C})$, bestehend aus allen Matrizen, die an der gleichen Stelle wie in $P_{x}$ Nullspalten haben. Ferner sei die Matrix $Q_{x}$ definiert als

$$
Q_{x}=I-P_{x} \quad(\varkappa=1, \ldots, k) .
$$


Man rechnet sofort nach, daß

$$
\left\{\begin{array}{l}
\text { a) } Q_{x} J Q_{x}=J Q_{x} \\
\text { b) } P_{1} J P_{1}=J P_{1}=P_{1} J .
\end{array} \quad(\varkappa=1, \ldots, k)\right.
$$

Das benutzen wir in

Hilfssatz 2. 21. a) $\boldsymbol{G}_{\mu \nu}\left(\mathfrak{U}_{x}\right)<\mathfrak{H}_{x}\left(\varkappa=1, \ldots, k ; \mu \geqq N^{\prime} ; \nu=0, \ldots, \mu\right)$

b) Die Einschränkung von $\boldsymbol{G}_{\mu \nu}$ auf $\mathfrak{U}_{1}$ hängt nur von dem einen Jordankästchen $J_{i}^{\tau} a b$.

Zum Beweis benutzen wir Satz 2. 1, zu dessen Anwendung wir $\mu$ mit $N$ bezeichnen. Zum Nachweis von a) seien $H_{0}, \ldots, H_{N-1} \in \mathfrak{U}_{x}$ beliebig vorgegeben.

Wir zeigen, daß für die durch (1) eindeutig bestimmte Folge $\left(H_{v}\right)_{\nu=0}^{\infty} \in \mathfrak{F}$ gilt:

$$
H_{v} \in \mathfrak{U}_{x} \quad \text { für alle } \nu \geqq N .
$$

Rechtsmultiplikation von (1) mit $Q_{x}$ liefert

$$
\sum_{\nu=0}^{\mu} B_{\mu-\nu}\left(H_{\nu} Q_{x}\right)-H_{\mu-s}\left(J Q_{x}\right)+(\mu-s) H_{\mu-s} Q_{x}=0 \quad(\mu \geqq N+s)
$$

und wegen (2.20) a):

$$
(+) \quad \sum_{\nu=0}^{\mu} B_{\mu-\nu}\left(H_{\nu} Q_{x}\right)-\left(H_{\mu-s} Q_{x}\right)\left(J Q_{x}+(\mu-s) I\right)=0 \quad(\mu \geqq N+s) .
$$

Daher ist die Folge $\left(H_{\nu} Q_{x}\right)_{\nu=0}^{\infty}$ Lösung von (1) mit $J Q_{x}$ statt $J$, wobei natürlich $\left|J Q_{x}\right| \leqq l$ und $\left(H_{\nu} Q_{x}\right)_{v=0}^{\infty} \in \mathfrak{F}$.

Aus der eindeutigen Lösbarkeit von $(+)$ in $\mathfrak{F}$ folgt aus

unmittelbar, daß alle weiteren

$$
H_{\nu} Q_{\star}=0 \quad(\nu=0, \ldots, N-1)
$$

$$
H_{\nu} Q_{x}=0 \quad(v \geqq N),
$$

also $H_{v} \in \mathfrak{U}_{x}$. Speziell $H_{N}$ hat die Darstellung

$$
H_{N}=\sum_{\nu=0}^{N-1} G_{N \nu} H_{\nu}
$$

und weil $H_{0}, \ldots, H_{N-1} \in \mathfrak{U}_{x}$ beliebig wählbar sind, folgt Teil a) für

$$
\mu \geqq N^{\prime}, \quad v=0, \ldots, \mu-1,
$$

und natürlich auch für $\boldsymbol{G}_{\mu \mu}=-I$.

Zum Beweis von b) nehmen wir $H_{0}, \ldots, H_{N-1} \in \mathfrak{U}_{1}$ an. Wie oben ergibt sich durch Rechtsmultiplikation von (1) mit $P_{1}$ unter Berücksichtigung von (2.20) b):

$$
(++) \quad \sum_{\nu=0}^{\mu} B_{\mu-\nu}\left(H_{\nu} P_{1}\right)-\left(H_{\mu-s} P_{1}\right)\left(J P_{1}+(\mu-s) I\right)=0 \quad(\mu \geqq N+s),
$$

wobei wieder $\left|J P_{1}\right| \leqq l$, und wegen a) für alle $v: H_{v} P_{1}=H_{v}$. Die gleiche Folge

$$
\left(H_{v}\right)_{v=0}^{\infty} \in \mathfrak{F}
$$

ist daher Lösung von (1) und $(++)$. Markiert man die Abhängigkeit der $G_{\mu \nu}$ von $J$ durch

so liefert Satz 2. 1 einerseits

$$
G_{\mu \nu}=: G_{\mu \nu}(J),
$$

$$
H_{N}=\sum_{\nu=0}^{N-1} G_{N v}(J) H_{v}
$$


andererseits wegen $(++)$

$$
H_{N}=H_{N} P_{1}=\sum_{\nu=0}^{N-1} G_{N v}\left(J P_{1}\right) H_{\nu}
$$

also hängt die Einschränkung $\operatorname{der} G_{N v}$ auf $\mathfrak{U}_{1}$ nur von $J P_{1}$ und damit nur von $J_{i}^{\tau}$ ab.

Bezeichnet man in Abhängigkeit von $J_{i}^{\tau}$

$$
P_{1}=: p^{i \tau} \quad\left(i=1, \ldots, m ; \tau=1, \ldots, t_{i}\right),
$$

wozu wir $P^{0,0}$ als die zur unteren $(n-r, n-r)$-Nullmatrix in $J$ gehörende Projektionsmatrix rechnen wollen, so folgt aus dem vorigen Hilfssatz durch einfache Rechnung:

Korollar 2. 22. Das System $\left(H_{v}\right)_{v=0}^{N+8-1}$ ist genau dann Lösung von (2.17), wenn, für alle $i=1, \ldots, m ; \tau=1, \ldots, t_{i}$ sowie für $(i, \tau)=(0,0),\left(H_{v} P^{i \tau}\right)_{v=0}^{N+1}$ Lösung von (2. 17).

Daher genügt es, (2.17) mit Systemen $\left(H_{y}\right)_{y=0}^{N+8-1}$ zu lösen, deren Koeffizienten alle in demselben zum Jordankästchen $J_{i}^{\tau}$ gehörenden Unterraum $\mathfrak{H}_{1}$ liegen. Für $H_{v} \in \mathfrak{U}_{1}$ sei das dem $J_{i}^{\tau}$ entsprechende Spaltensystem

$$
\left(h_{1 v}, \ldots, h_{k v}\right)
$$

dazu sei $H_{v}^{x}(\varkappa=1, \ldots, k)$ definiert durch das Spaltensystem

so daß also

$$
\left(0, \ldots, 0, h_{x \nu}, 0, \ldots, 0\right)
$$

$$
H_{v}=\sum_{x=1}^{k} H_{\nu}^{*} ; \quad \sum_{x=j}^{k} H_{\nu}^{*} \in \mathfrak{U}_{j} \quad(j=1, \ldots, k) .
$$

Um die $\mathrm{G}_{\mu \nu}$, auf $\mathfrak{U}_{1}$ eingeschränkt, weiter zu untersuchen, setzen wir wieder $\mu=N$; für $v=0,1, \ldots, N-1$ seien beliebige $H_{v} \in \mathfrak{U}_{1}$ vorgegeben. Für $H_{N}$ mit

$$
H_{N}=\sum_{\nu=0}^{N-1} G_{N \nu} H_{v}
$$

folgt nach Hilfssatz 2. 21, b) für $x=1, \ldots, k$

denn der Ausdruck

$$
H_{N}^{\varkappa}=\left(\boldsymbol{P}_{\kappa}-\boldsymbol{P}_{\kappa+1}\right) \sum_{\nu=0}^{N-1} G_{N \nu}\left(\sum_{j=1}^{\kappa} H_{\nu}^{j}\right) \quad\left(\text { mit } \boldsymbol{P}_{k+1}=0\right)
$$

liefert keinen Beitrag zu $H_{N}^{\kappa}$.

$$
G_{N v}\left(\sum_{j=x+1}^{k} H_{v}^{j}\right) \in \mathfrak{U}_{\kappa+1}
$$

Für die entsprechenden Spalten von $H_{N}$ gelten mit gewissen Matrizen $G_{N \nu}^{x i} \in M_{n}(\mathbb{C})$ die Gleichungen

$$
h_{x N}=\sum_{\nu=0}^{N-1} \sum_{j=1}^{x} G_{N \nu}^{x j} h_{j v} \quad(x=1, \ldots, k) .
$$

Aus der durch Satz 2: 1, (1) eindeutig bestimmten Folge $\left(H_{v}\right)_{v=0}^{\infty}$ ergibt sich als weitere Lösung von (1) die Folge mit den entsprechenden Spaltensystemen

$$
\left(0, h_{1 v}, \ldots, h_{k-1, v}\right) \quad(v=0,1,2, \ldots) .
$$

Darauf (2.23) angewandt, liefert einerseits

$$
h_{x-1, N}=\sum_{\nu=0}^{N-1} \sum_{j=2}^{x} G_{N \nu}^{x j} h_{j-1, v},
$$


während unmittelbar nach (2. 23)

$$
h_{\varkappa-1, N}=\sum_{\nu=0}^{N-1} \sum_{j=2}^{\varkappa} G_{N \nu}^{\varkappa-1, j-1} h_{j-1, v} .
$$

Da wir die $h_{x v}(v=0, \ldots, N-1)$ beliebig wählen können, ist

$$
G_{N, \nu}^{\varkappa-1, j-1}=G_{N \nu}^{\varkappa j} \quad(v=0, \ldots, N-1 ; \varkappa=2, \ldots, k ; j=2, \ldots, \varkappa) .
$$

Mit gewissen $G_{N v}^{\varkappa} \in M_{n}(\mathbb{C})(\varkappa=0, \ldots, k-1)$ schreiben wir daher

$$
G_{N \nu}^{\varkappa j}=: G_{N \nu}^{\varkappa-j} \text {. }
$$

Aus der Folge $\left(H_{\nu}\right)_{\nu=0}^{\infty}$ erhalten wir eine weitere Lösung von (1) in Satz 2. 1, wenn wir in jedem $H_{v}$ das Spaltensystem $\left(h_{1 v}, \ldots, h_{k v}\right)$, entsprechend verkürzt oder um Nullspalten erweitert, an die Stelle eines anderen Jordankästchens $J_{i}^{\sigma}$ zum gleichen Eigenwert $\lambda_{i}$ rücken. Damit sind die nach $(2.21)$, b) nur von $J_{i}^{\tau}$ abhängigen $G_{\mu \nu}^{\sigma}$ jetzt auch unabhängig von Lage und Größe des Jordankästchens. Das rechtfertigt die Bezeichnung

$$
G_{\mu \nu}^{\varkappa}:=G_{\mu \nu}^{\varkappa}\left(\lambda_{i}\right) \quad\left(\mu \geqq N^{\prime}, v=0, \ldots, \mu-1, \varkappa=0,1,2, \ldots\right) .
$$

Zusätzlich definieren wir

$$
\begin{aligned}
& G_{\mu \mu}^{0}(\lambda):=-I \\
& \left(\mu \geqq N^{\prime}, \lambda \in \mathbb{C}\right) \\
& G_{\mu \mu}^{\varkappa}(\lambda):=0 \\
& (x \geqq 1) \text {. }
\end{aligned}
$$

Als Ergebnis dieser Überlegungen fassen wir zusammen:

Satz 2. 24. Gemäß der Aufteilung von J zerfällt Gleichungssystem (2.17) in Gleichungen für die Spaltensysteme

$$
\left(h_{1 v}, \ldots, h_{k v}\right)_{v=0}^{N+8-1} \quad\left(k:=k_{i}^{\tau}\right),
$$

die in $\left(H_{\nu}\right)_{\nu=0}^{N+8-1}$ an der Stelle eines $J_{i}^{\tau}$ stehen. Diese Gleichungen lauten
a) $\sum_{\nu=0}^{\mu} B_{\mu-\nu} h_{x \nu}=0$
$(\varkappa=1, \ldots, k ; \mu=0, \ldots, s-1)$
b) $\sum_{\nu=0}^{\mu} B_{\mu-\nu} h_{1 \nu}-\left(\lambda_{i}+\mu-s\right) h_{1 \mu-s}=0$
c) $\left.\sum_{\nu=0}^{\mu} B_{\mu-\nu} h_{x \nu}-\left(\lambda_{i}+\mu-s\right) h_{x \mu-s}-h_{x-1 \mu-s}=0 \quad(x=2, \ldots, k)\right\}^{(\mu-s, \ldots-1)}$
d) $\sum_{j=1}^{x} \sum_{\nu=0}^{\mu} G_{\mu \nu}^{x-j}\left(\lambda_{i}\right) h_{j v}=0$
$(\varkappa=1, \ldots, k ; \mu=N, \ldots, N+s-1)$.

Die entsprechenden Spalten der durch (2.18) definierten $H_{\nu}$ sind bestimmt durch

$$
\sum_{j=1}^{x} \sum_{\nu=0}^{\mu} G_{\mu \nu}^{\varkappa-j}\left(\lambda_{i}\right) h_{j v}=0 \quad(\varkappa=1, \ldots, k ; \mu=N+s, N+s+1, \ldots) .
$$

In komplexen Koeffizienten ausgeschrieben, hat das homogene Gleichungssystem (2. 25) $k \cdot n \cdot s$ mehr Gleichungen als Unbekannte, d) besteht nämlich aus $k \cdot n \cdot s$ Gleichungen. Die Lösungsgesamtheit von a)-c) ist mit den Bezeichnungen vom 1. Kapitel gerade $\mathfrak{N}_{N+8-1}^{[k]}\left(\lambda_{i}\right)$; da für die Koeffizientenmatrizen gilt:

$$
\text { Rang von (2.25) } \leqq \text { Rang von (2.25), a)-c) }+k \cdot n \cdot s \text {, }
$$


so ist die Dimension der Lösungsgesamtheit von ganz (2.25) mindestens

$$
\operatorname{dim} \mathfrak{R}_{N+s-1}^{[k]}\left(\lambda_{i}\right)-k \cdot n \cdot s .
$$

Da in (2.19) $N+s-1 \geqq p+q+1$ vorausgesetzt war, berechnet man nach den Hilfssätzen 1. 29 und 1. 30

$$
\operatorname{dim} \mathfrak{R}_{N+s-1}^{[k]}\left(\lambda_{i}\right)=\operatorname{dim} \mathfrak{M}_{p}^{[k]}\left(\lambda_{i}\right)+k \cdot d .
$$

Zusammenfassend notieren wir

Hilfssatz 2. 26. Die Lösungsgesamtheit von (2. 25) hat als Dimension mindestens

$$
\operatorname{dim} \mathfrak{M}_{p}^{[k]}\left(\lambda_{i}\right)-k \cdot(n \cdot s-d)
$$

$\operatorname{Da} \mathfrak{M}_{p}^{[k]}\left(\lambda_{i}\right) \sim \mathfrak{R}_{q+p+1}^{[k]}\left(\lambda_{i}\right) / \hat{\mathfrak{N}}_{q+p+1}^{[k]}\left(\lambda_{i}\right)$, läßt sich $\operatorname{dim} \mathfrak{M}_{p}^{[k]}\left(\lambda_{i}\right)$ aus den Größen $t_{i}$ und $k_{i}^{\tau}$ in Satz 1. 32, $\gamma$ ) sofort ausrechnen. Mit den Beweismethoden von Satz 1. 19 sieht man sofort, daß

$$
\left\{\begin{array}{l}
\operatorname{dim} \mathfrak{M}_{p}^{[1]}\left(\lambda_{i}\right)=t_{i} \\
\operatorname{dim} \mathfrak{M}_{p}^{[\varkappa+1]}\left(\lambda_{i}\right)=\operatorname{dim} \mathfrak{M}_{p}^{[\varkappa]}\left(\lambda_{i}\right)+\#\left\{\tau \mid k_{i}^{\tau} \geqq \varkappa+1\right\} \quad(\varkappa=1,2,3, \ldots) .
\end{array}\right.
$$

Wir bezeichnen für $k=1,2, \ldots$

$$
w_{i}^{k}=\operatorname{dim} \mathfrak{M}_{p}^{[k]}\left(\lambda_{i}\right)-k \cdot(n \cdot s-d) .
$$

Ein Lösungssystem $\left(h_{1 v}, \ldots, h_{k v}\right)_{v=0}^{N+s-1}$ von (2.25) läßt sich nach Satz 2.16 und Satz 2. 24 eindeutig zu einer Lösung der Rekursionen (1.5) fortsetzen mit

$$
\sum_{\nu=0}^{\infty} x^{\nu} h_{x \nu}=: h_{x}(x), \quad \text { konvergent für } x \in \mathfrak{\Re}_{R} \quad(\varkappa=1,2, \ldots, k) .
$$

Nach (1.6) kann man der Folge $\left(h_{1 v}, \ldots, h_{k v}\right)_{v=0}^{\infty}$ eine in $\hat{\mathfrak{R}}_{R}$ analytische Lösung des Differentialgleichungssystems

$$
x^{s+1} y^{\prime}(x)-B(x) y(x)=0
$$

zuordnen, nämlich

$$
y(x)=x^{\lambda_{i}} \sum_{j=1}^{k} \frac{1}{(k-j) !}(\log x)^{k-j} h_{j}(x)
$$

Die weiteren in (1.6) erwähnten Lösungen von (4),

$$
(* *)
$$

$$
x^{\lambda_{i}} \sum_{j=1}^{x} \frac{1}{(x-j) !}(\log x)^{x-j} h_{j}(x)
$$$$
(\varkappa=1, \ldots, k-1)
$$

lassen sich natürlich in Gestalt $(*)$ mit dem gleichen $k$ schreiben und gehören zu den Lösungssystemen

$$
\left(0, \ldots, 0, h_{1 v}, \ldots, h_{x v}\right)_{v=0}^{N+8-1}
$$

von (2. 25). In diesem Sinne folgt aus Hilfssatz 2. 26 unmittelbar

Satz 2. 29. Falls $w_{i}^{k}>0$ für einen Index $i \in\{1, \ldots, m\}$ und eine natürliche Zahl $k$, so existieren mindestens $w_{i}^{k}$ linear unabhängige Lösungen von (4) der Form

$$
x^{\lambda_{i}} \sum_{j=1}^{k} \frac{1}{(k-j) !}(\log x)^{k-j} h_{j}(x)
$$

zur festen Potenz $\lambda_{i}$, mit $h_{j}: \mathfrak{R}_{R} \rightarrow \mathbb{C}^{n}$ holomorph $(j=1, \ldots, k)$. 
Um zur Formulierung für Matrizen zurückzukehren, wählen wir die $w_{i}^{k}$ linear unabhängigen Lösungen von (4) so, daß mit jeder Lösung $(*)$ sämtliche nichttrivialen Lösungen $(* *)$ aufgezählt sind: so erhalten wir $w_{i}^{k}$ Spalten einer Lösung von (1),

$$
Y(x)=H(x) x^{J},
$$

mit $J$ in Jordanscher Normalform, wobei die gesamte Spaltenzahl der Jordankästchen zu $\lambda_{i}$ genau $w_{i}^{k}$ beträgt. Das Verfahren wenden wir an auf alle Nullstellen $\lambda_{i}$ aus Satz 1. 32 $\alpha$ ), für die ein $w_{i}^{k}>0$ existiert; $k$ sei gewählt mit $w_{i}^{k}$ maximal.

Mit der Bezeichnung

$$
w_{i}=\max \left\{0, w_{i}^{k}: k \in \mathbb{N}\right\} \quad(i=1, \ldots, m)
$$

ist daher gezeigt:

Satz 2. 30. Für $r_{\max }^{\prime}$, den maximalen Rang einer Lösung von (1) der Gestalt

$$
Y(x)=H(x) x^{J} \quad \text { mit } \quad H: \mathfrak{\Re}_{R} \rightarrow M_{n}(\mathbb{C}) \text { holomorph, }
$$

gilt die Ungleichung

$$
\sum_{i=1}^{m} w_{i} \leqq r_{\max }^{\prime} \leqq \sum_{i=1}^{m} v_{i}
$$

Die 2. Ungleichung gilt, weil $Y(x)$ notwendig formale Lösung ist. - Abschließend notieren wir

Satz 2. 31. Die Differentialgleichung (1) besitzt eine Fundamentallösung der Form

$$
Y(x)=H(x) x^{J}, \quad H(x)=\sum_{\nu=0}^{\infty} x^{\nu} H_{v} \quad\left(x \in \mathfrak{R}_{R}\right)
$$

genau dann, wenn

$$
d=n \cdot s .
$$

Besveis. Eine Fundamentallösung der Form (2), für die wir ohne Einschränkung die Bedingungen (A), (B) und (C) aus dem 1. Kapitel annehmen dürfen, hat auch als formale Lösung den Rang $n$, folglich ist nach Satz 1. 39 notwendig $d=n \cdot s$.

Falls umgekehrt $d=n \cdot s$, besitzt nach Hilfssatz 2. 26 die Lösungsgesamtheit von (2. 25) mindestens die Dimension von $\mathfrak{M}_{p}^{[k]}\left(\lambda_{i}\right)$. Andererseits liegt jede Lösung von (2. 25), da man sie zu einer Lösung von (1.5) fortsetzen kann, schon in $\mathfrak{M}_{N+8-1}^{[k]}\left(\lambda_{i}\right)$, wobei

$$
\operatorname{dim} \mathfrak{M}_{p}^{[k]}\left(\lambda_{i}\right)=\operatorname{dim} \mathfrak{M}_{N+8-1}^{[k]}\left(\lambda_{i}\right)
$$

(siehe dazu $(\gamma)$ im Beweis von Hilfssatz 1. 30). Folglich ist der Lösungsraum von (2. 25) genau $\mathfrak{M}_{N+8-1}^{[k]}\left(\lambda_{i}\right)$. Nach Hilfssatz 1.20 liefert $\mathfrak{M}_{N+8-1}^{[k]}\left(\lambda_{i}\right)$ eindeutig alle formalen Lösungen von (1.5), nach Satz 2.16 und Satz 2. 24 eindeutig die konvergenten Lösungen. Es folgt

Korollar 2. 32. Falls $d=n \cdot s$, ist jede formale Lösung der Form (2) schon konvergent.

Darüber hinaus liefert Satz 1. 39 die Existenz einer formalen Lösung vom Rang $n$, was den Beweis dieses Satzes beschließt.

Unmittelbare Folgerung ist der bei D. A. Lutz [8] als Satz 1 angegebene

Satz. Falls $S: \mathfrak{A}_{R} \rightarrow M_{n}(\mathbb{C})$ holomorph, mit 0 als mindestens $n \cdot s$-facher Nullstelle, so hat die gestörte Differentialgleichung

$$
x^{8+1} Y^{\prime}(x)=(B(x)+S(x)) Y(x)
$$

genau dann ein regulär-singuläres Fundamentalsystem, wenn (1) dieses besitzt. 
Unser Beweis liegt in der Tatsache, $\operatorname{daß} q \leqq n \cdot s-1$, so daß sich $A_{q}(\lambda)$ und damit $d_{q}=d$ beim Ubergang von $B(x)$ auf $B(x)+S(x)$ nicht ändern.

Das von D. A. Lutz in der gleichen Arbeit angegebene Kriterium, wann genau eine regulär-singuläre Fundamentallösung von (1) vorliegt, lautet:

Satz 3 ([8]). Es sei die Folge $\left(\mathfrak{U}_{v}\right)_{v=0}^{\infty}$ rekursio definiert durch

$$
\mathfrak{A}_{0}(x)=I, \mathfrak{A}_{v+1}(x)=\mathfrak{A}_{\nu}^{\prime}(x)+x^{-(8+1)} \mathfrak{A}_{\nu}(x) B(x) ;
$$

es bezeichne $p\left(\mathfrak{A}_{v}\right)$ die Ordnung von Null als Pol von $\mathfrak{A}_{\nu}$. Dann gilt

(1) hat eine regulär-singuläre Fundamentallösung genau dann, wenn

$$
p\left(\mathfrak{A}_{v}\right) \leqq v+(n-1) \cdot s \quad(\nu=n, n+1, \ldots, N),
$$

wobei $N$ von $B(x)$ abhängig ist.

Der entscheidende Nachteil dieses Kriteriums ist, daß eine obere Schranke für $N$ nicht allgemein angegeben wird. $N$ läßt sich zwar im Einzelfall auf rein algebraischem Wege bestimmen, jedoch ist das Verfahren dazu recht kompliziert. Auch ist an diesem Kriterium seine Abhängigkeit nur von höchstens den ersten $n \cdot s$ Koeffizienten von $B(x)$ nicht mehr unmittelbar einzusehen. Siehe hierzu Zusatz bei der Korrektur.

\section{Beispiele und Anwendungen}

3. 1. Satz von Lettenmeyer. Der von F. Lettenmeyer [5] 1926 gefundene Satz lautet:

Satz. Im Differentialgleichungssystem

$$
x^{s_{i}} \eta_{i}^{\prime}(x)=\sum_{j=1}^{n} \alpha_{i j}(x) \eta_{j}(x) \quad(i=1, \ldots, n)
$$

seien die $\alpha_{i j}$ komplexwertige Funktionen, holomorph für $x \in \mathfrak{\Re}_{R}$, die $s_{i}$ nichtnegative ganze Zahlen mit

$$
0<\sum_{i=1}^{n} s_{i}=\sigma<n
$$

Dann besitzt (3.1) mindestens $n-\sigma$ linear unabhängige in $\mathfrak{A}_{R}$ holomorphe Lösungen.

Zum Beweis bringen wir mit

$$
s+1=\max \left\{s_{i} \mid i=1, \ldots, n\right\}
$$

(3.1) auf Form (4), wobei wir $s=0$ zulassen müssen. Da wir nur holomorphe Lösungen suchen, fallen die Úberlegungen des 1. Kapitels zur Bestimmung von $J$ weg: wir setzen $J=0$ und bestimmen $N \in \mathbb{N}$ so, daß Satz 2.1 anwendbar wird. Notwendige und hinreichende Bedingung für eine holomorphe Lösung von (3.1),

$$
h(x)=\sum_{\nu=0}^{\infty} x^{\nu} h_{v}
$$

ist Gleichungssystem (2.25) mit $k=1, \lambda_{i}=0$ für $\left(h_{v}\right)_{v=0}^{N+8-1}$. Die Koeffizientenmatrix von (2. 25), a), b) in diesem Fall ist $A_{N+8-1}(0)$, c) fällt wegen $k=1$ weg, und d) besteht aus $n \cdot s$ Gleichungen mit zunächst unbekannten Koeffizienten; im Fall $s=0$ fällt d) weg, wie wir am Ende von Kapitel 2. 1. bemerkt haben. Wie die folgende Rechnung lehrt, besitzt $A_{N+8-1}(0)$ mindestens $n \cdot(s+1)-\sigma$ Nullzeilen. Weil Gleichungssystem (2. 25) $n \cdot s$ mehr Gleichungen als Unbekannte hat, wird damit die Dimension der Lösungsgesamtheit mindestens $n-\sigma$, folglich existieren $n-\sigma$ holomorphe Lösungen von (3.1). 
Zum Abzählen der Nullzeilen in $A_{N+8-1}(0)$ bezeichne

$$
v_{\mu}=\text { Anzahl der Indizes } i \text { mit } s_{i}=\mu \quad(\mu=0, \ldots, s+1) .
$$

Es ist die $i$-te Zeile in

$$
\left(B_{j} B_{j-1} \cdots B_{0} 0 \cdots 0\right) \quad(j=0, \ldots, s)
$$

sicher dann Null, wenn $s_{i}<s+1-j$, also $s_{i} \leqq s-j$, daher die Anzahl der Nullzeilen in $\left(B_{j} \cdots B_{0} 0 \cdots 0\right)$ mindestens

$$
\sum_{\mu=0}^{s-j} v_{\mu}=n-\sum_{\mu=8-j+1}^{s+1} v_{\mu}
$$

Insgesamt wird die Mindestzahl von Nullzeilen in $A_{N+s-1}(0)$

$$
(s+1) n-\sum_{j=0}^{8} \sum_{\mu=8-j+1}^{s+1} v_{\mu}=(s+1) n-\sum_{\mu=1}^{s+1} \mu v_{\mu}=(s+1) n-\sigma
$$

was den Beweis vollendet.

3. 2. Ein Zahlenbeispiel. Zur Erläuterung der im 1. Kapitel gefundenen Methoden betrachten wir das System

$$
x^{2}\left(\begin{array}{l}
\eta_{1}^{\prime} \\
\eta_{2}^{\prime} \\
\eta_{3}^{\prime} \\
\eta_{4}^{\prime}
\end{array}\right)=\left(\begin{array}{cccc}
x & 1 & -x^{2} & 0 \\
0 & x & 4 x^{3} & x^{2} \\
0 & 0 & 3 x & 1 \\
x & 0 & 0 & x
\end{array}\right)\left(\begin{array}{l}
\eta_{1} \\
\eta_{2} \\
\eta_{3} \\
\eta_{4}
\end{array}\right) .
$$

Offensichtlich ist der Satz von Lettenmeyer hier nicht mehr anwendbar. Um zu prüfen, ob ein regulär-singuläres Fundamentalsystem vorliegt, berechnen wir $d$, indem wir die ersten $A_{v}(\lambda)$ notieren und ihre Defekte als Matrizen über $\mathbb{C}(\lambda)$ bestimmen. Da stets $q \leqq n s-1$ (nach (1.24)), beschränken wir uns auf $A_{3}(\lambda)$, welches ja $A_{0}(\lambda), A_{1}(\lambda)$ und $A_{2}(\lambda)$ als Teilmatrizen enthält.

$A_{3}(\lambda)$ hat folgende Gestalt, - die leeren Felder sind Nullen:



Eine notwendige Bedingung für das Vorliegen einer Fundamentallösung ist erfüllt, da $B_{0}$ nilpotent. Es ergibt sich

$$
d_{0}=2 ; \quad d_{1}=3 ; \quad d_{2}=4 .
$$


Damit ist

$$
q=2, \quad d=n \cdot s
$$

folglich liegt ein regulär-singuläres Fundamentalsystem vor. - Zum Beweis, daß $d_{1}=3$, beachte man, daß die 6 . Zeile ein Polynom-Vielfaches der 1 . Zeile ist; $d_{2}=4$ gilt, weil die 10. Zeile Linearkombination mit Polynomkoeffizienten aus der 3., 5. und 8. Zeile ist, nämlich

$$
Z_{10}=\left(1-\lambda(1-\lambda)^{2}\right) Z_{3}-Z_{5}+\lambda(1-\lambda) Z_{8}
$$

Diese Tatsache ergänzen wir durch eine analoge Aussage für die Spalten:

die 11. Spalte ist $(\lambda-1) \times$ letzte Spalte,

die 7. Spalte ist Linearkombination mit Polynomkoeffizienten der 9., 12. und 14. Spalte.

Um die Lösungen der Differentialgleichung zu berechnen, benötigen wir $\chi(\lambda)$, den g.g.T. aller 12-zeiligen Unterdeterminanten von $A_{3}(\lambda)$. Wir hatten gesehen, daß für jedes feste $\lambda$ die 2., 4., 6. und 10. Zeile Linearkombinationen der übrigen Zeilen sind, und analoges für die 7., 11., 13. und 15. Spalte gilt.

Bezeichnet nun $\Delta(\lambda)$ die Determinante, entstanden aus $A_{3}(\lambda)$ durch Streichen der genannten Zeilen und Spalten, so gilt

daher ist

$$
\forall \lambda \in \mathbb{C}: \operatorname{rg} A_{3}(\lambda)<12 \curvearrowleft \Delta(\lambda)=0
$$

$$
\chi(\lambda)=\text { const. } \Delta(\lambda) .
$$

Mehrfache Entwicklung von $\Delta(\lambda)$ nach Zeilen oder Spalten liefert

$$
\chi(\lambda)=\left|\begin{array}{rrrc}
3-\lambda & 0 & 1 & 0 \\
-1 & -\lambda & 0 & 1 \\
0 & 1 & -\lambda & 0 \\
4 & 0 & 1 & -\lambda-1
\end{array}\right|=\lambda^{2}(\lambda+1)(\lambda-3)
$$

und damit die einfachen Nullstellen -1 und 3 sowie 0 als doppelte Nullstelle von $\chi$.

Von den in Satz 1.32 aufgezählten Größen sind zunächst

$$
m=1, \quad \lambda_{1}=-1, \quad p=4 ;
$$

aus $d=n \cdot s$ folgt wegen Satz 1. 39

Man rechnet sofort nach, daß

$$
v_{1}=4
$$

folglich ist

$$
\operatorname{det} A_{7}(-1)=-7=d+3
$$

und wegen $v_{1}=4$

$$
\operatorname{dim} \mathfrak{M}_{4}^{[1]}(-1)=3
$$

$$
\operatorname{dim} \mathfrak{M}_{4}^{[2]}(-1)=4=\operatorname{dim} \mathfrak{M}_{4}^{[k]}(-1) \quad \text { für alle } k \geqq 2 .
$$

Daher liegen 3 linear unabhängige logarithmenfreie Lösungen und eine mit $\log x$ in der 1. Potenz vor.

In diesem Fall interessiert die Ordnung der Stelle $x=0$ als Nullstelle bzw. Pol der logarithmenfreien Lösungen: Da die Zahl 3 einfache Nullstelle von $\chi$ ist, mit maxi- 
malem Realteil, ist $\operatorname{dim} \mathfrak{M}_{0}^{[k]}(3)=1$ für alle $k$; und Hilfssatz 1.30, angewandt auf $\lambda_{0}=3, k=1$ liefert genau eine holomorphe Lösung $y_{1}$ mit Null als dreifacher Nullstelle; hier wird

Wir rechnen weiter nach, daß

$$
y_{1}(x)=\left(\begin{array}{c}
0 \\
x^{5} \\
x^{3} \\
0
\end{array}\right)
$$

folglich ist

$$
\operatorname{det} A_{4}(0)=4=d+1
$$

$$
\operatorname{dim} \mathfrak{M}_{0}^{[1]}(0)=1 \text {. }
$$

Nimmt man nun eine Basis von $\mathfrak{M}_{4}^{[1]}(-1)$ wie im Beweis von Hilfssatz 1. 35 an, sieht man wegen

daß auch

$$
\operatorname{dim} \mathfrak{M}_{4}^{[1]}(-1)=3
$$

$$
\operatorname{dim} \mathfrak{M}_{0}^{[1]}(-1)=1 \text {; }
$$

und es muß je eine logarithmenfreie Lösung zu den Potenzen 0 und -1 geben. Eine Berechnung aus den Rekursionen liefert

$$
y_{2}(x)=\left(\begin{array}{c}
0 \\
x^{2} \\
1 \\
-3 x
\end{array}\right), \quad y_{4}(x)=\left(\begin{array}{c}
4 \\
-3 x \\
x^{-1} \\
-4
\end{array}\right)
$$

Zur Bestimmung der logarithmenbehafteten Lösung folgern wir aus den Überlegungen des Hilfssatz 1. 35, daß wegen

sicher

$$
\operatorname{dim} \mathfrak{M}_{0}^{[2]}(3)=\operatorname{dim} \mathfrak{M}_{0}^{[2]}(-1)=1
$$

$$
\operatorname{dim} \mathfrak{M}_{0}^{[2]}(0)=2
$$

und daß die Lösung mit $\log x$ zur Potenz 0 gehört. Die Rekursionen liefern hier

$$
y_{3}(x)=\log x \cdot y_{2}(x)+\left(\begin{array}{c}
-3 x \\
0 \\
0 \\
x
\end{array}\right)
$$

3. 3. Einordnung der Differentialgleichung $n$-ter Ordnung. Die komplexe Differentialgleichung $n$-ter Ordnung

$$
\begin{array}{cc}
x^{s+n} \eta^{(n)}(x)-\sum_{i=0}^{n-1} x^{i} q_{n-i}(x) \eta^{(i)}(x)=0 & \text { mit } s \in \mathbb{N} \\
q_{i}: \mathfrak{\Re}_{R} \rightarrow \mathbb{C} \text { holomorph }(i=1, \ldots, n), & \text { nicht alle } q_{i}(0)=0,
\end{array}
$$

ist mit gewissen in $\mathfrak{I}_{R}$ holomorphen $\hat{q}_{i}$, die nicht sämtlich in Null verschwinden, sowie mit dem Operator

äquivalent der Gleichung

$$
\delta=x \frac{d}{d x}
$$

$$
x^{s} \delta^{n} \eta(x)-\sum_{i=0}^{n-1} \hat{q}_{n-i}(x) \delta^{i} \eta(x)=0 .
$$


$\mathrm{Zu}$ einem System aus $n$ Lösungen $\left(\eta_{1}(x), \ldots, \eta_{n}(x)\right)$ von (3. 3') ist die Matrix

Lösung von

$$
Y(x)=\left(\delta^{\nu-1} \eta_{i}(x)\right)_{(n, n)} \quad \text { mit }\left(\delta^{\nu-1} \eta_{i}(x)\right)_{v=1}^{n} \text { als } i \text {-ter Spalte }
$$

$$
x^{s+1} Y^{\prime}(x)-B(x) Y(x)=0
$$

mit

$$
B(x)=\left(\begin{array}{ccc}
0 & x^{s} & 0 \\
1 & & 0 \\
0 & 0 & 1 \\
\hat{q}_{n}(x) & \cdots & \hat{q}_{1}(x)
\end{array}\right)
$$

Nimmt man $Y(x)$ in der Gestalt an

$$
Y(x)=H(x) x^{J} \quad \text { mit } H: \mathbb{R}_{R} \backslash\{0\} \rightarrow M_{n}(\mathbb{C}) \text { holomorph, }
$$

so verhalten sich die Lösungen $\eta_{i}$ von (3.3) bei Annäherung an $x=0$ bestimmt genau dann, wenn $H$ in 0 höchstens einen Pol besitzt bzw. bei geeigneter Wahl von $J$ in ganz $\mathfrak{\pi}_{R}$ holomorph ist. Damit ist (3.3) auf unsere allgemeine Fragestellung zurückgeführt, wobei $B(x)$ in Gestalt (3.4) vorliegt.

Mit den Potenzreihendarstellungen der $\hat{q}_{i}(x)$ für $x \in \mathfrak{\Re}_{R}$

$$
\hat{q}_{i}(x)=\sum_{\nu=0}^{\infty} x^{\nu} \beta_{n+1-i, \nu} \quad(i=1, \ldots, n)
$$

haben die $B_{v}$ die Gestalt

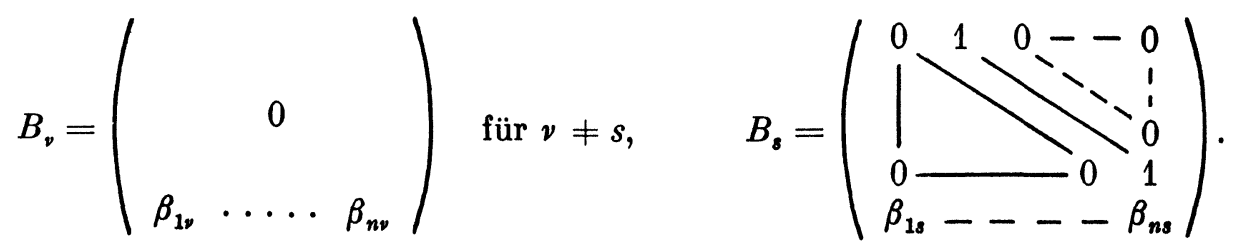

Da nach Voraussetzung die letzte Zeile in $B_{0}$ nicht verschwindet, ist für $v=0,1, \ldots, s-1$

$$
d_{v}=(\nu+1)(n-1) \text {. }
$$

Zur Berechnung von $d_{s}$ und $\chi(\lambda)$ betrachten wir




Offensichtlich sind die nichtverschwindenden Zeilen (über dem Körper $\mathbb{C}(\lambda)$ ) linear unabhängig, daher

Es folgt

$$
d_{s}=s \cdot(n-1)=d_{s-1} \text {. }
$$

$$
\left\{\begin{array}{l}
q=s-1 \\
d=s \cdot(n-1) .
\end{array}\right.
$$

Für ein festes komplexes $\lambda$ ist $\delta_{s}(\lambda)<d$ genau dann, wenn die nichtverschwindenden Zeilen von $A_{s}(\lambda)$ - als Matrix über $\mathbb{C}$ - linear abhängig sind.

Das ist damit äquivalent, daß

$$
\operatorname{det}\left(\begin{array}{cccc}
\beta_{10} & \beta_{20} & \cdots & \beta_{n 0} \\
-\lambda & 1 & 0 \\
0 & 0
\end{array} \mid=0\right.
$$

denn die restlichen nichtverschwindenden Zeilen aus $A_{s}(\lambda)$ sind bezüglich der ersten $n$ Komponenten linear unabhängig. Die Determinante hat den Wert

$$
f_{0}(\lambda)=\beta_{10}+\beta_{20} \lambda+\cdots+\beta_{n 0} \lambda^{n-1}
$$

daher gilt

$$
\chi(\lambda)=\text { const. } f_{0}(\lambda)
$$

Ähnliche Überlegungen, auf die Matrizen $A_{s+\mu}(\lambda)$ angewandt, liefern für alle $\mu \in \mathbb{N}_{0}$

$$
\chi_{\mu}(\lambda)=\text { const. } \cdot \prod_{\nu=0}^{\mu} f_{0}(\lambda+v) .
$$

Falls $f_{0}(\lambda) \neq$ const., gilt für die Vielfachheiten der in Satz 1.32 ausgezeichneten Nullstellen $\lambda_{i}$ von $\chi$ als Nullstellen in $\chi_{p}$ :

$$
v_{i}=\sum_{\nu=0}^{p}\left(\text { Vielfachheit von } \lambda_{i}+v \text { als Nullst. in } f_{0}\right)
$$

und nach Hilfssatz 1. 33:

$$
r_{\max }=\text { Grad von } f_{0} \leqq n-1 .
$$

Schon die in (3.6) erwähnte Tatsache, daß $d<n \cdot s$, liefert mit Satz 2.31 einen direkten Beweis für den Satz, daß für $s>0$ die Differentialgleichung (3.3) kein Fundamentalsystem von Lösungen, die sich bei $x=0$ bestimmt verhalten, besitzt.

Aus (3.9) folgern wir die stärkere Aussage, die Thomé [1, a] mit Reduktion der Differentialgleichung und Induktion bewiesen hat:

Satz 3. 10. Die Zahl der linear unabhängigen, sich bei Null bestimmt verhaltenden Lösungen von (3.3) ist höchstens so groß wie der Grad von $f_{0}$.

Hier liegt übrigens ein gutes Anschauungsbeispiel für Satz 1.38 mit $d<n s$ vor: es ist nämlich

$$
\operatorname{Grad} f_{0}=n-1 \curvearrowright \beta_{n 0} \neq 0
$$


und letzteres bedeutet genau, daß

$$
\omega_{0}=n-1,
$$

in Übereinstimmung mit Satz 1. 38, 2.

Um jetzt auf die Rekursionen näher einzugehen, nehmen wir eine formale Lösung von (1) mit $B(x)$ gemäß (3.4) an,

$$
Y(x)=\sum_{\nu=0}^{\infty} H_{\nu} x^{\nu I+J}
$$

unter den Annahmen (A), (B) und (C) aus dem 1. Kapitel. Die Spalten der $H_{\nu}$, die zurn Jordankästchen $J_{i}^{\tau}$ der Spaltenzahl $k$ und zum Eigenwert $\lambda$ gehören, seien

$$
h_{1 v}, \ldots, h_{k v} \quad(v=0,1,2, \ldots),
$$

die Komponenten von $h_{x v}$ seien

speziell bezeichne

$$
h_{x \nu}^{\alpha} \quad(\alpha=1, \ldots, n),
$$

$$
\gamma_{x v}:=h_{x \nu}^{1} \quad\left(x=1, \ldots, k ; \nu \in \mathbb{N}_{0}\right) .
$$

Nach (1.6) sind im Falle der Reihenkonvergenz die Funktionen

$$
\eta_{x}(x)=x^{\lambda} \sum_{j=1}^{x} \frac{1}{(\varkappa-j) !}(\log x)^{x-j} \sum_{\nu=0}^{\infty} x^{\nu} \gamma_{j v} \quad(x=1, \ldots, k)
$$

Lösungen von (3. 3); in jedem Fall nennen wir diese Reihen formale Lösungen von (3. 3).

Von den Rekursionen (1.5), die von den $h_{x y}$ gelöst werden, notieren wir die letzte Zeile jeder Gleichung. Es ergibt sich

$$
\begin{array}{cr}
\text { a) } \sum_{\nu=0}^{\mu}\left(\sum_{i=1}^{n} \beta_{i \mu-\nu} h_{x v}^{i}\right)=0 \quad(\mu=0, \ldots, s-1 ; \varkappa=1, \ldots, k) \\
\text { b) } \sum_{\nu=0}^{\mu}\left(\sum_{i=1}^{n} \beta_{i \mu-\nu} h_{1 \nu}^{i}\right)-(\lambda+\mu-s) h_{1 \mu-8}^{n}=0 \quad(\mu=s, s+1, \ldots) \\
\text { c) } \sum_{\nu=0}^{\mu}\left(\sum_{i=1}^{n} \beta_{i \mu-\nu} h_{x \nu}^{i}\right)-(\lambda+\mu-s) h_{x \mu-8}^{n}-h_{x-1 \mu-8}^{n}=0 \\
(\varkappa=2, \ldots, k ; \mu=s, s+1, \ldots) .
\end{array}
$$

Die ersten $n-1$ Zeilen jeder Gleichung (1.15)a) liefern keinen Beitrag; aus Gleichung (1. 15), b) für die Nummer $\mu+s$ entnehmen wir:

und daher

$$
h_{1 \mu}^{i+1}-(\lambda+\mu) h_{1 \mu}^{i}=0 \quad(i=1, \ldots, n-1)
$$

$$
h_{1 \mu}^{i}=(\lambda+\mu)^{i-1} \gamma_{1 \mu} \quad(i=1, \ldots, n ; \mu=0,1,2, \ldots) .
$$

Mit den Definitionen

$$
\left\{\begin{array}{l}
f_{v}(\lambda)=\sum_{i=1}^{n} \beta_{i v} \lambda^{i-1} \quad(\nu \neq s) \\
f_{s}(\lambda)=-\lambda^{n}+\sum_{i=1}^{n} \beta_{i s} \lambda^{i-1}
\end{array}\right.
$$

wobei $f_{0}$ schon unter (3.7) definiert war, ergeben sich durch Einsetzen von $\left(3.13^{\prime}\right)$ in (3. 12), a) und b) für die $\gamma_{1 v}$ die Rekursionen

$$
\sum_{\nu=0}^{\mu} f_{\mu-v}(\lambda+v) \gamma_{1 v}=0 \quad(\mu=0,1,2, \ldots) .
$$


Aus den jeweils ersten $n-1$ Zeilen der Gleichungen (1.15), c) für $\mu+s$ folgt

$$
h_{x \mu}^{i+1}=(\lambda+\mu) h_{x \mu}^{i}+h_{x-1, \mu}^{i} \quad\left(i=1, \ldots, n-1 ; \varkappa=2, \ldots, k ; \mu \in \mathbb{N}_{0}\right),
$$

woraus man mit einem Induktionsbeweis leicht sieht, daß

$$
h_{x \mu 1}^{i+1}=\sum_{j=0}^{x-1}\left(\begin{array}{l}
i \\
j
\end{array}\right)(\lambda+\mu)^{i-j} \gamma_{x-j, \mu}=\sum_{j=0}^{x-1} \frac{1}{j !} \gamma_{x-j, \mu} \frac{\partial^{j}}{\partial \lambda^{j}}(\lambda+\mu)^{i} .
$$

Nimmt man (3.13') hinzu, haben wir stets

$$
h_{x \mu}^{i}=\sum_{j=0}^{x-1} \frac{1}{j !} \gamma_{x-j, \mu} \frac{\partial^{j}}{\partial \lambda^{j}}(\lambda+\mu)^{i-1} \quad\left(\mu \in \mathbb{N}_{0}, \varkappa=1, \ldots, k, i=1, \ldots, n\right)
$$

Dies für $x \geqq 2$ in $(3.12), c)$ eingesetzt, liefert in Verallgemeinerung von $\left(3.15^{\prime}\right)$ :

$$
\sum_{j=0}^{x-1} \frac{1}{j !} \sum_{\nu=0}^{\mu} f_{\mu-\nu}^{(j)}(\lambda+v) \gamma_{x-j, v}=0 \quad(\varkappa=1, \ldots, k ; \mu=0,1,2, \ldots) .
$$

Da sich die $h_{j \mu}^{i}$ für $i=2, \ldots, n$ durch (3.13) eindeutig aus den $\gamma_{j \mu}$ berechnen lassen, notieren wir

Satz 3. 16. Die Reihen (3.11) sind genau dann formale Lösungen von (3.3), wenn die Rekursionen

$$
\sum_{j=0}^{x-1} \frac{1}{j !} \sum_{\nu=0}^{\mu} f_{\mu-\nu}^{(j)}(\lambda+v) \gamma_{x-j, \nu}=0
$$

für $\varkappa=1, \ldots, k ; \mu=0,1,2, \ldots$ erfüllt sind.

Selbstverständlich braucht man (3.15) nur für die $\lambda_{i}$ aus Satz 1. 32 zu lösen; für $\mu \geqq p$ sind die Gleichungen (3.15) wegen $f_{0}\left(\lambda_{i}+\mu\right) \neq 0$ nach $\gamma_{x \mu}$ eindeutig auflösbar; wegen

$$
-f_{0}\left(\lambda_{i}+\mu\right) \gamma_{x \mu}=\sum_{j=1}^{x} \frac{1}{j !} \sum_{\nu=0}^{\mu} f_{\mu \rightarrow v}^{(j)}\left(\lambda_{i}+v\right) \gamma_{x-j, \nu}+\sum_{v=0}^{\mu-1} f_{\mu-v}\left(\lambda_{i}+v\right) \gamma_{x v}
$$

ist $\gamma_{x \mu}$ aus den $\gamma_{j v}$ mit $j=0, \ldots, x-1 ; \nu=0, \ldots, \mu$ sowie den $\gamma_{x \nu}$ mit $\nu=0, \ldots, \mu-1$ eindeutig bestimmbar. Daher notieren wir

Hilissatz 3. 17. Für jedes $\lambda_{i}$ aus Satz 1.32 und $k \in \mathbb{N}$ ist $\mathfrak{M}_{p}^{[k]}\left(\lambda_{i}\right)$ isomorph der Lösungsgesamtheit des Systems

$$
\sum_{j=0}^{x-1} \frac{1}{j !} \sum_{\nu=0}^{\mu} f_{\mu \rightarrow}^{(j)}\left(\lambda_{i}+v\right) \gamma_{x-j, \nu}=0 \quad(x=1, \ldots, k ; \mu=0, \ldots, p) .
$$

Zum Beweis zeigen wir, $\operatorname{da} B \mathfrak{M}_{p}^{[k]}\left(\lambda_{i}\right)$ die Gesamtheit aller $\left(h_{x v}\right)_{\substack{x=1, \ldots, k \\ v=0, \ldots, p}}$ ist, deren Komponenten $h_{x \nu}^{1}=\gamma_{x \nu}$ den Gleichungen (3.17) genügen und für deren übrige Komponenten gilt:

$$
\begin{aligned}
h_{x \nu}^{\alpha}=\left.\sum_{j=0}^{x-1} \frac{1}{j !} \gamma_{x-j, \nu} \frac{\partial^{j}}{\partial \lambda^{j}}(\lambda+\nu)^{\alpha-1}\right|_{\lambda=\lambda_{i}} \\
\\
(v=0, \ldots, p ; \varkappa=1, \ldots, k ; \alpha=2, \ldots, n) .
\end{aligned}
$$

Offensichtlich ist (3.18) und (+) dafür notwendig, daß man $\left(h_{\substack{x v\\)_{x=0, \ldots, \ldots, k} \\ v=0, \ldots}}\right.$ zu einer Lö-

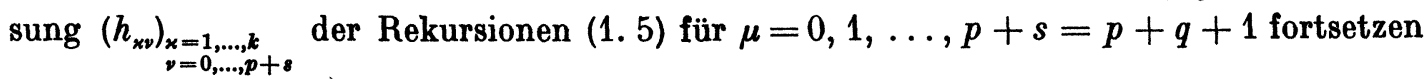
kann, denn (+) folgt aus (1.5) für $\mu=s, s+1, \ldots, p+s$. Nach den vorhergehenden Uberlegungen liegt umgekehrt jede Lösung von (3.18) und $(+)$ in $\mathfrak{M}_{p}^{[k]}\left(\lambda_{i}\right)$. 
Als hinreichende Bedingung für die Existenz (nicht mehr nur formaler) regulärsingulärer Lösungen von (3.3) notieren wir nun zusammenfassend

Satz 3. 19. Es sei $k \in \mathbb{N}$ beliebig, $\lambda_{i}$ eine der Nullstellen von $f_{0}$ mit

$$
\begin{aligned}
& \lambda_{i}-z \text { nicht Nullstelle von } f_{0} \text { für alle } z \in \mathbb{N}, \\
& p=\text { maximale ganzzahlige Differenz von Nullstellen von } f_{0} .
\end{aligned}
$$

Außerdem besitze das System (3.18) $l_{k}$ linear unabhängige Lösungen mit $l_{k}>k \cdot s$.

Dann existieren mindestens $l_{k}-k s$ linear unabhängige Lösungen von (3. 3) der Form

$$
x^{\lambda_{i}} \sum_{j=1}^{k} \frac{1}{(k-j) !}(\log x)^{k-j} \gamma_{j}(x)
$$

mit $\gamma_{j}: \mathfrak{A}_{R} \rightarrow \mathbb{C}$ holomorph.

Dieser Satz folgt aus Hilfssatz 2. 26 und Satz 2. 29 unmittelbar, da

$$
\operatorname{dim} \mathfrak{M}_{p}^{[k]}\left(\lambda_{i}\right)=l_{k} \quad \text { und } \quad d=(n-1) \cdot s .
$$

In seiner Arbeit [3] über dieses Problem hat Perron den Satz 3.16 vollständig und den Satz 3. 19 für $k=1$ bewiesen; letzterer entspricht seinen Ausführungen auf Seite 21 in [3]. Bei Behandlung der Fälle $k \geqq 2$, also der logarithmenbehafteten Lösungen (ebd. S. 23-26) ist Perron ein Fehler unterlaufen. Die dort auf S. 25 oben durchgeführte Differentiation nach $\lambda$ (bei Perron $\varrho$ ) ist nicht motiviert, entsprechend ist der Ausdruck unter (63) - in dem wir hier $e$ durch $s, N$ durch $N+2 s$, mit der Bedeutung aus Satz 2. 1 ersetzen - :

$$
\text { (+) } \quad \sum_{i=0}^{N+8-1} \gamma_{i} \frac{\partial y_{i}}{\partial \lambda}=\sum_{\nu=0}^{\infty}\left(\sum_{i=0}^{N+8-1} \gamma_{i} \frac{d D_{\nu}^{(i)}}{d \lambda}+\log x \sum_{i=0}^{N+8-1} \gamma_{i} D_{\nu}^{(i)}\right) x^{\lambda+\nu}
$$

nicht die allgemeine Gestalt einer Lösung mit $\log x$. - Man kann die angegebenen Größen $D_{v}^{(i)}$ nämlich so wählen, daß die Abschnitte $\left(D_{v}^{(i)}(\lambda)\right)_{v=0}^{N+s-1}(i=0, \ldots, N+s-1)$ gerade die konstanten Einheitsvektoren sind, dann würde der logarithmenfreie Teil in $(+)$ erst mit $x^{\lambda+N+8}$ beginnen, bei beliebig großem $N$. Entsprechend ist die auf S. 25 bei Perron angegebene Matrix zur Richtigstellung zu ersetzen durch

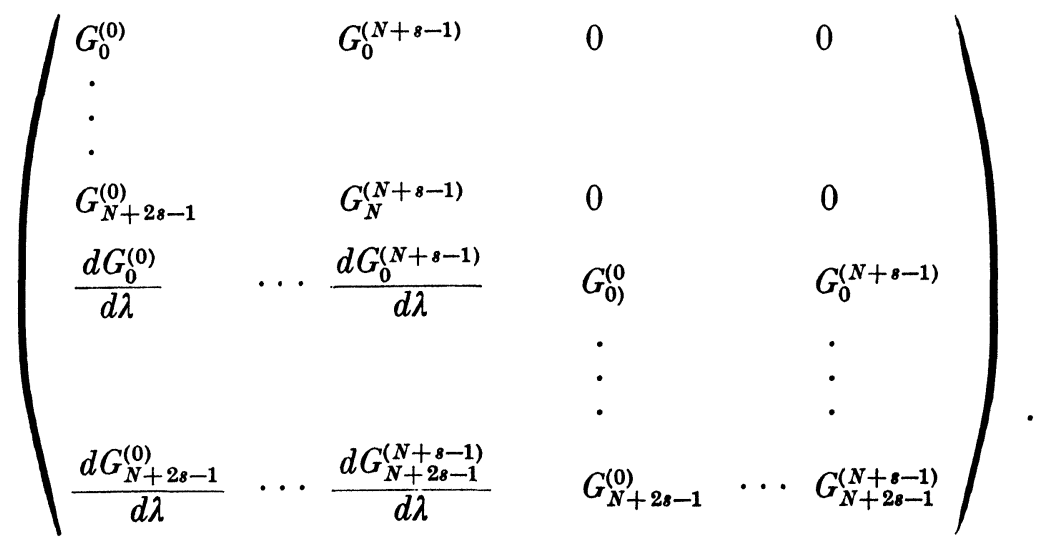

Dazu bemerken wir, daß mit Wahl der $\left(D_{v}^{(i)}(\lambda)\right)_{v=0}^{N+8-1}$ als Einheitsvektoren gilt:

$$
\begin{array}{ll}
G_{\nu}^{(i)}(\lambda)=f_{v-i}(\lambda+i) & \text { für } v=0, \ldots, N+s-1 ; i=0, \ldots, \nu, \\
G_{v}^{(i)}(\lambda)=0 & \text { für } v=0, \ldots, N+s-1 ; i \geqq v+1,
\end{array}
$$

während die $G_{v}^{(i)}$ für $v=N+s ; \ldots, N+2 s-1$ aus Grenzprozessen zu gewinnen sind. 
Läßt man die $2 s$ Zeilen mit den $G_{v}^{(i)}$ für $v \geqq N+s$ weg, erhält man die Koeffizientenmatrix von (3.18) mit $k=2$ und $N+s-1$ statt $p$, so daß sich nunmehr Satz 3.19 für $k=2$ ergibt.

\section{Schlußbemerkungen}

Es bleibt als offene Frage, ob ein Beweis des am Ende des 2. Kapitels zitierten Satzes von D. A. Lutz mit den Methoden dieser Arbeit möglich ist: vielleicht läßt sich die dort angegebene Zahl $N$ dann abschätzen.

Ein weiteres Problem sind Fundamentallösungen der Differentialgleichung (1) von allgemeinerer Gestalt, nämlich

$$
Y(x)=H(x) x^{J} e^{Q(x)}
$$

mit

$H$ holomorph in einem Sektor $\mathfrak{S}<\mathfrak{\Omega}^{R}$,

$$
\begin{aligned}
& H(x) \sim \sum_{v=0}^{\infty} x^{\frac{\nu}{p}} H_{v} \quad(\text { asymptotisch) mit } p \in \mathbb{N}, \\
& Q(x) \text { Diagonalmatrix, Polynom in } x^{\frac{1}{p}}, \\
& J \in M_{n}(\mathbb{C}) \text { konstant. }
\end{aligned}
$$

Die zugehörige Theorie ist z. B. in dem Buch von W. Wasow [11] dargestellt. Die Methoden des 1. Kapitels unserer Arbeit werden sich auch zur Gewinnung formaler Lösungen der Gestalt (*) anwenden lassen.

Zusatz bei der Korrektur. Der Wert von $N$ ist von W. B. Jurkat und D. A. Lutz, On the Order of Solutions of analytic Differential Equations, Proc. London Math. Soc. (3) 22 (1971), 465-482, zu $N=(n-1)(2 n s-1)$ angegeben. Dieses Ergebnis war dem Autor bei Einreichen der Arbeit nicht bekannt.

\section{Literatur}

[1] L. W. Thomé, Zur Theorie der linearen Differentialgleichungen, Journal reine angew. Math. 74 (1872), 193-217; 75 (1873), 265-291; 76 (1873), 273-302.

[2] H. v. Koch, Sur les intégrales régulières des équations différentielles linéaires, Acta Mathematica 16 (1892).

[3] O. Perron, Über diejenigen Integrale linearer Differentialgleichungen, welche sich an einer Unbestimmtheitsstelle bestimmt verhalten, Math. Annalen 70 (1911), 1-32.

[4] E. Hilb, Uber diejenigen Integrale linearer Differentialgleichungen, welche sich an einer Unbestimmtheitsstelle bestimmt verhalten, Math. Annalen 82 (1921), 40-41.

[5] F. Lettenmeyer, Über die an einer Unbestimmtheitsstelle regulären Lösungen eines Systems homogener linearer Differentialgleichungen. Sitzungsberichte Bayr. Akademie der Wiss., München 1926, 287-307.

[6] W. A. Harris, Y. Sibuya and L. Weinberg, Holomorphic Solutions of Lin. Diff. Systems at Singular Points, Arch. for Rat. Mech. and Anal. 35 (1969), 245-249.

[7] D. A. Lutz, On Systems of Linear Diff. Equations Having Regular-Singular Solutions, Journal of Diff. Equations 3 (1967), 311-322.

[8] D. A. Lutz, Some characterisations of systems of lin. diff. equations having regular-singular solutions, Transactions of AMS 126 (1967), 427-441.

[9] Gantmacher, Matrizenrechnung I. Berlin 1958, S. $120 \mathrm{ff}$.

[10] Coddington-Levinson, Theory of Ordinary Differential Equations, New York 1955.

[11] W. Wasow, Asymptotic Expansions for Ordinary Differential Equations, New York 1965.

Fachbereich Mathematik der Universität, 84 Regensburg 2, Postfach 